\title{
Changes in milk composition in obese rats consuming a high-fat diet
}

\author{
C. J. Bautista ${ }^{1}$, S. Montaño ${ }^{2}$, V. Ramirez ${ }^{3}$, A. Morales $^{1}$, P. W. Nathanielsz ${ }^{4}$, N. A. Bobadilla ${ }^{3,5}$ and \\ E. Zambrano ${ }^{1 *}$ \\ ${ }^{1}$ Departamento de Biología de la Reproducción, Instituto Nacional de Ciencias Médicas y Nutrición "Salvador Zubirán", \\ Mexico City, 14080, Mexico \\ ${ }^{2}$ Departamento de Nutrición Animal, Instituto Nacional de Ciencias Médicas y Nutrición "Salvador Zubirán”, Mexico City, \\ 14080 Mexico \\ ${ }^{3}$ Departamento de Nefrología y Metabolismo Mineral, Instituto Nacional de Ciencias Médicas y Nutrición "Salvador Zubirán", \\ Mexico City, 14080 Mexico \\ ${ }^{4}$ Department of Animal Science, University of Wyoming, Laramie, WY 82071-3684, USA \\ ${ }^{5}$ Instituto de Investigaciones Biomedicas, Universidad Nacional Autónoma de México, Mexico City, 04510 Mexico
}

(Submitted 9 March 2015 - Final revision received 10 September 2015 - Accepted 12 October 2015 - First published online 26 November 2015)

\section{Abstract}

Maternal obesity programmes offspring development. We addressed maternal obesity effects induced by high-fat diets on maternal mammary gland (MG) structure and function and offspring brain, liver and fat outcomes. Mothers were fed control (C, $n 5)$ or obesogenic (MO, $n 5)$ diet from the time they were weaned through pregnancy beginning at $120 \mathrm{~d}$, through lactation. At offspring postnatal day (PND) 20 , milk leptin and nutrients were determined. At the end of lactation, maternal liver and MG fatty acid profile were measured. Desaturase ( $\Delta 6 \mathrm{D}$ and $\Delta 5 \mathrm{D})$ and elongase (ELOVL 5 and ELOVL 2) protein was measured by immunohistochemistry and Western blotting (WB) in the liver and WB in the MG. In mothers, liver, MG and milk fat content were higher in MO than in C. Liver arachidonic acid (AA) and EPA and MG EPA were lower in MO than in C. Liver desaturases were higher in MO. The MG was heavier in MO than in C, with decreased $\Delta 5 \mathrm{D}$ expression in MO. Desaturases and elongases were immunolocalised in parenchymal cells of both groups. Milk yield, water, carbohydrate content, EPA and DHA were lower, whereas milk leptin and AA were higher in MO than in C. At PND 21 and 36, brain weight was less and fat depots were greater in MO offspring than in C. MO decreased male absolute brain weight but not female absolute brain weight. In conclusion, maternal obesity induced by an obesogenic diet negatively affects maternal liver and MG function with the production of significant changes in milk composition. Maternal obesity adversely affects offspring metabolism and development.

Key words: Maternal obesity: Mammary gland development: Maternal liver metabolism: Milk composition: Offspring development

Obesity and over-nutrition together constitute an ever-increasing world health problem ${ }^{(1)}$. Further maternal over-nutrition has been demonstrated to result in offspring metabolic programming ${ }^{(2,3)}$, in multiple organ systems, for example, pancreas and liver ${ }^{(2)}$, as well as in central and peripheral nervous systems involved in energy homoeostasis $^{(4-7)}$. Human epidemiological ${ }^{(2,8)}$ and experimental animal studies $^{(9-12)}$ have shown a correlation between maternal diet and milk composition. Few studies address the mechanisms by which maternal obesity induced by a high-fat diet regulates liver and mammary gland (MG) differentiation and function during lactation and the implications for the observed changes in milk synthesis and composition ${ }^{(13)}$. Long-chain PUFA (LC-PUFA) are essential for normal neonatal mammalian development. Arachidonic acid (AA, 20:4n-6) and DHA (C22:6n-3) are essential for neuronal developmental and cognitive function ${ }^{(14-16)}$. EPA $(20: 5 n-3)$ is the precursor of several eicosanoid products that are necessary for normal function of the immunological system $^{(17,18)}$. The concentration of these lipids, AA and EPA $=2 \%$ and $\mathrm{DHA}=0 \cdot 17 \%$, in rat milk fat is minimal ${ }^{(17,19)}$. Milk is by far the greatest source of these fatty acids for the neonate, as enzymatic activity of desaturases $\Delta 6 \mathrm{D}$ and $\Delta 5 \mathrm{D}$ and elongases ELOVL 5 and 2 is low in early life and the fetus and neonate cannot produce the required amounts of LC-PUFA ${ }^{(20,21)}$.

We hypothesised that a maternal obesity (MO) phenotype resulting from consumption of a high-fat diet during growth, gestation and lactation induces (1) alterations in maternal liver metabolism; (2) variations in MG differentiation and function; (3) adverse changes in maternal milk nutrient concentration;

Abbreviations: AA, arachidonic acid; C, control; LC-PUFA, long-chain PUFA; MG, mammary gland; MO, maternal obesity; PND, postnatal day; WB, Western blotting.

*Corresponding author: E. Zambrano, email zamgon@unam.mx 
and (4) programmes offspring liver, fat and brain development in a sex-dependent manner.

\section{Methods}

To ensure homogeneity of mothers studied in the different experimental groups, female albino Wistar rats were obtained exclusively from Instituto Nacional de Ciencias Médicas y Nutrición Salvador Zubirán (INCMNSZ), Mexico. All procedures were approved by INCMNSZ Animal Experimentation Ethics Committee. Animals were held in American Association for Accreditation of Laboratory Animal Care. We studied female rats $\mathrm{F}_{0}$ fed control (C, control $n$ 5) (laboratory chow Zeigler Rodent RQ22-5) or obesogenic diet (MO, $n$ 5) (Table 1) during growth (21-120 d of life) and pregnancy and lactation, as previously described in detail $^{(22)}$. Female rats were mated at $120 \mathrm{~d}$ of age. Mothers delivering litters over fourteen or under ten were excluded from the experiment. To ensure $F_{1}$ offspring homogeneity, on postnatal day (PND) 2, all $F_{1}$ litters studied were adjusted to ten pups with equal numbers of male and female pups whenever possible. A fixed amount of fresh food was provided daily. During lactation, the food remaining after $24 \mathrm{~h}$ was weighed daily ${ }^{(22)}$. Offspring were weaned at PND 21, housed five per cage and fed the control diet.

\section{Measurement of milk composition}

Milk was obtained at PND 20. Food and pups were removed from mothers at 06.00 hours. After $4 \mathrm{~h}, 0.8 \mathrm{U}$ oxytocin (ip) was administered to the mother and milk expressed 15 min later and quantified as total milk yield (ml). Milk samples were vortexed, divided into aliquots and frozen at $-20^{\circ} \mathrm{C}$ until analysis. Milk samples were thawed at $37^{\circ} \mathrm{C}$ and vortexed before pipetting to ensure sample uniformity. Milk water was studied by gravimetric analysis. Milk carbohydrate concentration was determined by spectrophotometry (absorbance $492-550 \mathrm{~nm}$ ) using the enzymatic glucose oxidase method (AccuTrack). Protein concentration was determined by the Bradford assay (Bio-Rad). Milk leptin concentration was determined by RIA, as previously described ${ }^{(11)}$, using a commercial rat kit (Linco Research Inc.) with a detection limit of $0.5 \mathrm{ng} / \mathrm{ml}$. Milk samples were assayed in duplicate.

Table 1. Maternal diet composition

(Percentages)

\begin{tabular}{lcc}
\hline Components (\%) & C (\%) & MO (\%) \\
\hline Protein & 22 & 23.5 \\
Polysaccharide & 31 & 21 \\
Simple sugars & 31 & 21 \\
Fibre & 4 & 5 \\
Minerals & 6 & 5 \\
Vitamins & 1 & 1 \\
Fat & 5 (maize oil) & 25 (20 lard +5 maize oil) \\
$\quad$ SFA & 0.8 & 8.7 \\
Monosaturates & 1.0 & 9.6 \\
$\quad$ PUFA & 3.2 & 6.4 \\
Energy (kJ/g) & 16.7 & 20.5 \\
Energy (kcal/g) & 4.0 & 4.9 \\
\hline
\end{tabular}

C, control group; MO, maternal obesity group.

\section{Maternal liver and mammary gland collection}

At PND 21, after a 4-h fast, mothers were rapidly euthanised by decapitation by experienced personnel trained in the use of the rodent guillotine (Thomas Scientific), and trunk blood was collected into polyethylene tubes, allowed to clot at $4^{\circ} \mathrm{C}$ for $1 \mathrm{~h}$, centrifuged at $1500 \mathrm{~g}$ for $15 \mathrm{~min}$ at $4^{\circ} \mathrm{C}$ and serum was stored at $-20^{\circ} \mathrm{C}$ until assayed. Maternal liver ${ }^{(23)}$ and MG chain were excised and weighed ${ }^{(24)}$. The right inferior liver lobule was fixed for morphometric analysis and immunohistochemistry (IHC), whereas the left inferior liver lobule was immediately frozen at $-75^{\circ} \mathrm{C}$ for protein analysis by Western blotting (WB). The remainder of the liver was collected at $-20^{\circ} \mathrm{C}$ for Folch analysis and fatty acid profile. The MG beneath the 6th right nipple (counted from the cephalad end) was sectioned longitudinally into two halves and immediately immersion-fixed in $4 \%$ paraformaldehyde in neutral PBS. After $24 \mathrm{~h}$ of fixation, tissue sections were dehydrated with ethanol at increasing concentrations from 75 to $95 \%$ and embedded in paraffin. Sections $(5 \mu \mathrm{m})$ were stained with haematoxylin-eosin. The MG beneath the 4 th and 5 th right nipples was frozen at $-20^{\circ} \mathrm{C}$ for Folch analysis and fatty acid profile. The MG below the 4th, 5 th and 6th left nipples was immediately frozen at $-75^{\circ} \mathrm{C}$ for $\mathrm{WB}$ analysis $^{(24)}$.

\section{Morphometric analysis}

The percentage area stained for liver fat was evaluated at $100 \times$ magnification.

For each animal, ten MG pictures were analysed containing at least 150 lobules/rat at 10X. Area was expressed as the percentage of adipose and parenchymal tissues (acinar and ductal epithelium). In all, fifty acini per animal were measured at higher magnification $(100 \times)$, and results were expressed as acini area; nucleus and cytoplasm area for cells in each acini (approximately 7-15 cells/ acinus) were measured and results were expressed as cytoplasm and nuclei size. All procedures were evaluated using the AxioVision software $^{(24)}$. All histological measurements were performed by two independent observers without knowledge of the source of the tissues, and the results were averaged.

\section{Western blot analysis of protein concentration}

A section of liver tissue and MG were homogenised in RIPA lysis buffer (PBS 1\%, NP-40 1\%, sodium deoxicolate $0.5 \%$, SDS $0.1 \%$ and sodium azide $0.006 \%(\mathrm{w} / \mathrm{v}))$. The total lysate protein concentration was determined by the Bradford method. Protein samples were separated on $10 \%$ SDS-PAGE gels and transferred onto a polyvinylidene fluoride membrane $(0.45 \mu \mathrm{m}$ Millipore). Each membrane was probed with antibodies from Santa Cruz biotechnology either to $\Delta 6 \mathrm{D}$ (sc-98480), $\Delta 5 \mathrm{D}$ (sc-101953), ELOVL 5 (sc-374138) or ELOVL 2 (sc-54874) at a 1:500 dilution in Tris-buffered saline Tween (20 mm TrisHCl, $500 \mathrm{~mm}-\mathrm{NaCl}, \mathrm{pH} 7 \cdot 4$, $0.01 \%$ Tween-20) with 5\% non-fat dried milk, and incubated for $60 \mathrm{~min} . \Delta 6 \mathrm{D}$ and $\Delta 5 \mathrm{D}$ were incubated with goat anti-rabbit IgG-horseradish peroxidase (sc-2004) secondary antibodies and ELOVL 5 and 2 with donkey anti-goat IgG-HRP (sc-2020). Proteins were detected by chemiluminescence (Millipore). Images were captured by an E3 biochemical imaging system (UVP) and spot 
densitometry analysis was performed using the vision work system software (UVP). The first four spots of each gel contained samples from the $\mathrm{C}$ group and the next four spots contained samples from the MO group. A total of eight samples were included per gel. All results were normalised to $\beta$-actin as the loading control ${ }^{(24)}$.

\section{Immunohistochemistry analysis}

Liver and MG paraffin sections $(5 \mu \mathrm{m})$ were immunostained with Santa Cruz Biotechnology; rabbit polyclonal $\Delta 6 \mathrm{D}$ (sc-98480) and $\Delta 5 \mathrm{D}$ (sc-101953), and goat polyclonal ELOVL 5 (sc-374138) and ELOVL 2 (sc-54874) 1:200 dilution, ABC Elite kit, Vector Laboratories and visualised using $2.5 \%$ nickel sulphate with $0.02 \%$ DAB (3,3'-diaminobenzidine tetrahydrochloride) chromogen in $0.175 \mathrm{~m}$-sodium acetate. Cell counts were performed on an Olympus BX51 light microscope using image analysis software (Image-Pro Plus, version 3.1; Media Cybernetics Inc.). For the data analyses of protein in the liver by IHC, we used the image software as previously described ${ }^{(3)}$. For MG, the IHC was used only for immunolocalisation, as the distribution of lobules and fat was very different between control and MO mothers.

\section{Fatty acid analysis}

Maternal liver, MG, milk and offspring liver lipids were extracted by a modified Folch technique. Samples were homogenised with $2 \mathrm{ml}$ of $0.9 \% \mathrm{NaCl}$ and $5 \mathrm{ml}$ of chloroform-methanol (2:1), as previously described ${ }^{(23,24)}$. Fatty acid extraction was performed by the addition of chloroform $(3 \times 2 \mathrm{ml})$. The organic phase was pooled and 120-150 $\mu$ l of methanol was added until the organic phase turned transparent, and then $1 \mathrm{~g}$ of $\mathrm{Na}_{2} \mathrm{SO}_{4}$ was added and vortexed to provide the residue for analysis. The organic phase was evaporated under a stream of $\mathrm{N}_{2}$.

\section{Preparation of fatty acid methyl esters}

Samples for fatty acid methyl esters (FAME) were prepared as previously described ${ }^{(24)}$. Briefly, $2 \mathrm{ml}$ of methanol, $100 \mu \mathrm{l}$ of toluene and $40 \mu \mathrm{l}$ of $2 \%$ methanolic sulphuric acid were added to the above residue and heated at $90^{\circ} \mathrm{C}$ for $2 \mathrm{~h}$. Tubes were then placed on ice, and $1 \mathrm{ml}$ of $5 \% \mathrm{NaCl}$ was added. FAME were extracted with hexane $(3 \times 2 \mathrm{ml})$, and the mixture was centrifuged at $1500 \boldsymbol{g}$ for $1 \mathrm{~min}$. The organic phase was pooled and evaporated under a stream of $\mathrm{N}_{2}$. Hexane $(200 \mu \mathrm{l})$ was added to the residue, which was then centrifuged at $1500 \boldsymbol{g}$ for $5 \mathrm{~min}$. The clear solution was injected in an Agilent model 6850 GC equipped with a flame ionisation detector. Automatic split injection was carried out using an Agilent 6850 auto sampler. The chromatographic column was an HP-INNOWax capillary column ( $30 \mathrm{~m}, 0.25 \mathrm{~mm}, 0.25 \mathrm{~m}$ ) (J \& W Scientific). One hundred twenty-five micrograms of heptadecanoic acid was added to $100 \mathrm{mg}$ of tissue as an internal standard. A 1- $\mu$ l sample was injected in split mode $(50: 1)$ at $250^{\circ} \mathrm{C}$. The carrier gas was $\mathrm{He}_{2}$ with a constant linear velocity of $24 \mathrm{~cm} / \mathrm{s}$, and the interface temperature was maintained at $280^{\circ} \mathrm{C}$. The oven temperature was raised from 50 to $230^{\circ} \mathrm{C}$. Identification of the FAME was based upon retention times obtained for methyl ester standards from PolyScience, and each one was expressed as a percentage of total fatty acid in the sample.

\section{Quantification of TAG}

Liver TAG were extracted using the Folch method and quantified with a colorimetric (absorbance $546 \mathrm{~nm}$ ) commercial kit from RANDOX CE ${ }^{\circledR}$ (RX MONZA Method GPO-PAP). Briefly, $10 \mu \mathrm{l}$ of lipids was diluted in $1000 \mu \mathrm{l}$ of enzyme reagent and incubated for $10 \mathrm{~min}$ at $20-25^{\circ} \mathrm{C}$. All samples were assayed in duplicate.

\section{Offspring parameters at postnatal day 21 and 36}

After weaning, all offspring were fed chow diet. Offspring body weight was determined at random in two male and two female $\mathrm{F}_{1}$ pups/litter per age ( $n 5$ litters). At PND 21 and 36, pups were fasted for $4 \mathrm{~h}$ and euthanised by decapitation. Offspring adipose tissue from visceral and retroperitoneal areas was collected and weighed together with the brain and liver.

\section{Statistical analysis}

Statistical analysis was performed using unpaired Student's $t$ test with $P$ set at $<0 \cdot 05$. Results from WB were normalised with $\beta$-actin and compared between groups. Offspring data from the same litter were averaged for analysis to provide 5 litters per group. Preliminary analysis for differences according to the sex of the pup at PND 21 revealed no difference, and thus all data at this age were pooled. At PND 36, the results were expressed by sex. All data are presented as mean values with their standard errors.

\section{Results}

\section{Maternal parameters at postnatal day 21}

Maternal body weight in the $\mathrm{C}$ and MO groups ( $\mathrm{C}=352$ (SEM 6) $\mathrm{g}$ and $\mathrm{MO}=356$ (SEM 14) g) at PND 21 were similar. However, total body adipose tissue weight was higher in $\mathrm{MO}$ than in $\mathrm{C}$ mothers $(\mathrm{C}=4$ (SEM 0.2) $\mathrm{g}$ and $\mathrm{MO}=19$ (SEM 3) $\mathrm{g} ; P<0 \cdot 01$ ). Using average data for the lactation period, food intake $(\mathrm{C}=63$ (SEM 2.6) $\mathrm{g}$ and $\mathrm{MO}=50(\operatorname{sem} 2 \cdot 8) \mathrm{g} / \mathrm{d})$ and energy intake $(\mathrm{C}=1054(\mathrm{SEM} 42) \mathrm{kJ} / \mathrm{d}$ $(\mathrm{C}=252(\mathrm{SEM} \mathrm{10}) \mathrm{kcal} / \mathrm{d})$ and $\mathrm{MO}=1025(\mathrm{SEM} 59) \mathrm{kJ} / \mathrm{d}(\mathrm{MO}=245$ (SEM 14) $\mathrm{kcal} / \mathrm{d})$ ) were similar between groups. Fat intake was higher in $\mathrm{MO}$ than in $\mathrm{C}(\mathrm{C}=3.2($ SEM 0.2$) \mathrm{g} / \mathrm{d}$ and $\mathrm{MO}=12.5$ (SEM 0.7$) \mathrm{g} / \mathrm{d} ; P<0.05$ ).

\section{Maternal liver analysis at postnatal day 21}

Liver weight was similar in both groups, whereas the percentage of liver fat was higher in MO than in $\mathrm{C}$ (Table 2). The percentages of AA and EPA were lower in MO than in C, whereas DHA was similar in the two groups (Fig. 1(a), (b) and (c)). Finally, the hepatic fatty acid profile exhibited more SFA and monosaturates and less $n-3$ and $n-6$ PUFA in MO than in C (Table 2).

\section{Maternal liver $\Delta 6 D, \Delta 5 D$, ELOVL 5 and 2 protein expression by Western blotting and immunohistochemistry at postnatal day 21}

Hepatic $\Delta 6 \mathrm{D}$ and $\Delta 5 \mathrm{D}$ protein abundance and the percentage of immunostained tissue area were higher in MO than in $\mathrm{C}$ mothers (Fig. 2(a) and (b)). ELOVL 5 was similar in both groups (Fig. 2(c)). 
ELOVL 2 immunostaining was lower in $\mathrm{MO}$ than in $\mathrm{C}$, but protein levels by WB were similar in the two groups (Fig. 2(d)).

\section{Mammary gland weight and morphometric analysis at postnatal day 21}

At PND 21 MG weight, total fat by Folch and percentage of fat by histological analysis were higher in MO than in C (Table 2 and Fig. 3(d)). Histological analysis showed smaller acini and cytoplasm area and nuclei size in MO than in C (Fig. 3(a), (b), (c) and (e)). The percentages of AA and DHA were similar between groups (Fig. 1(d) and (f)), whereas EPA was lower in MO than in

Table 2. Maternal liver and mammary gland (MG) parameters at postnatal day (PND) 21

(Mean values with their standard errors; $n 5$ )

\begin{tabular}{|c|c|c|c|c|}
\hline \multirow[b]{2}{*}{ Groups } & \multicolumn{2}{|c|}{$\mathrm{C}$} & \multicolumn{2}{|c|}{ MO } \\
\hline & Mean & SEM & Mean & SEM \\
\hline \multicolumn{5}{|c|}{ Liver parameters at PND 21} \\
\hline Liver weight (g) & 15 & 0.4 & 15 & 0.8 \\
\hline Total liver fat (\%) & 6.9 & 0.26 & $12 \cdot 4^{\star}$ & 0.48 \\
\hline Liver fat (\% area) & 0.49 & 0.13 & $7 \cdot 13^{*}$ & 1.08 \\
\hline SFA & 40 & 3 & $49^{\star}$ & 4 \\
\hline Monosaturates & 16 & 1 & $29^{*}$ & 3 \\
\hline$n-3$ PUFA & 19 & 1 & $10^{*}$ & 2 \\
\hline$n-6$ PUFA & 24 & 2 & $11^{*}$ & 3 \\
\hline \multicolumn{5}{|l|}{ MG at PND 21} \\
\hline MG weight (g) & 21 & 2 & $29^{\star}$ & 2 \\
\hline Total MG fat (\%) & $9 \cdot 2$ & 0.5 & $13 \cdot 4^{*}$ & 0.4 \\
\hline MG fat (\% area) & 29 & 3 & $54^{*}$ & 1 \\
\hline SFA & 39 & 2 & $52^{\star}$ & 3 \\
\hline Monosaturates & 24 & 2 & 27 & 2 \\
\hline$n-3$ PUFA & 13 & 1 & $11^{*}$ & 1 \\
\hline$n-6$ PUFA & 24 & 2 & $13^{*}$ & 1 \\
\hline
\end{tabular}

C, control group; $\mathrm{MO}$, maternal obesity group.

* Significantly different compared with $C(P \leq 0.05)$.
C (Fig. (1)). Fatty acid analysis showed more SFA and less $n$-3 and n-6 PUFA in MO than C mothers (Table 2).

\section{Maternal mammary gland $\Delta 6 D, \Delta 5 D, E L O V L 5$ and 2 protein expression by Western blotting and immunohistochemistry localisation at postnatal day 21}

$\Delta 6 \mathrm{D}$, ELOVL 5 and ELOVL 2 protein expression by WB were similar in $\mathrm{C}$ and $\mathrm{MO}$ (Fig. 4). However, $\Delta 5 \mathrm{D}$ was lower in MO than in C (Fig. 4(b)). $\Delta 6 \mathrm{D}, \Delta 5 \mathrm{D}$, ELOVL 5 and ELOVL 2 were immunolocalised in parenchymal cells of both groups (Fig. 4).

\section{Milk composition at postnatal day 20}

Milk production and milk percentage water, carbohydrate content, EPA, DHA, n-3 PUFA and SFA were lower (Fig. 5(a), (b), (c), (h), (i) and (j)), whereas milk leptin, total fat, AA, monosaturates and fatty acids were higher in MO than in $\mathrm{C}$ (Fig. 5(e), (f), (g) and (j)). The percentages of protein and $n-6$ PUFA were similar in the two groups (Fig. 5(d) and (j)).

\section{Pup development at postnatal day 21 and 36}

Pup body weights were similar in the two groups at birth as were pup body and liver weights at PND 21 (Table 3). However, pup weight gain during lactation was greater in MO. Body weight at PND 36 was similar between groups in both sexes, but both $\mathrm{C}$ and MO male pups weighed more than female pups. Absolute adipose tissue weight and adipose tissue weight relative to body weight was greater in $\mathrm{MO}$ pups than in $\mathrm{C}$ at PND 21 and 36. Adipose tissue weight in male pups was greater than in female pups at PND 36 in both groups. Brain weight at PND 21 was less in MO compared with C, but when expressed
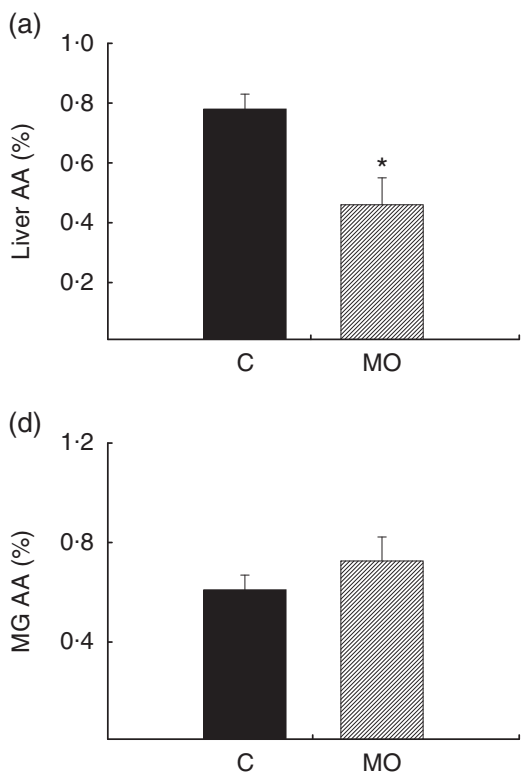

(b)

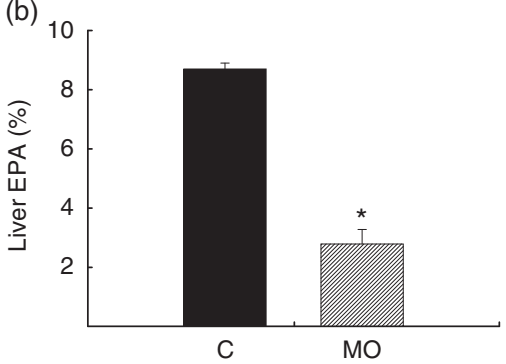

(e)

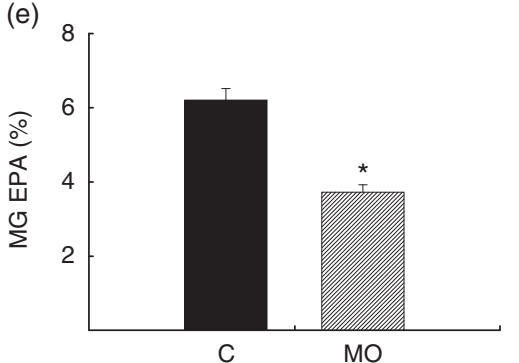

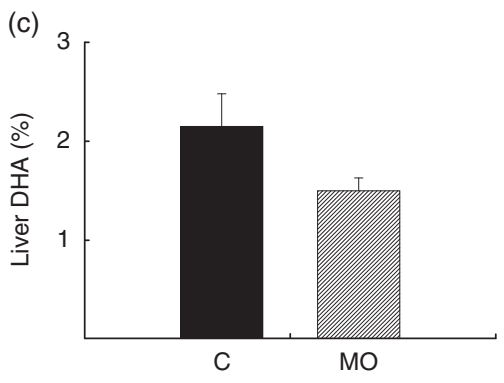

(f)

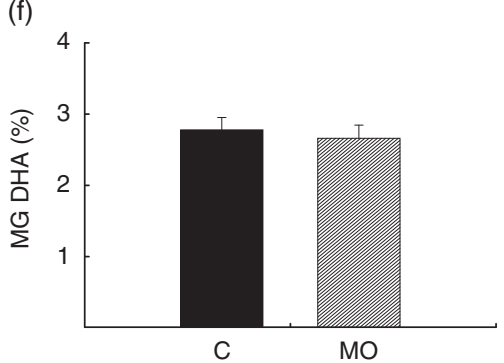

Fig. 1. Maternal liver at postnatal day (PND) 21. (a) Arachidonic acid (AA, \%), (b) EPA (\%) and (c) DHA (\%), and maternal mammary gland (MG) at PND 21, (d) AA (\%), (e) EPA (\%) and (f) DHA (\%). Values are means ( $n$ 5), with their standard errors represented by vertical bars. * Mean value was significantly different

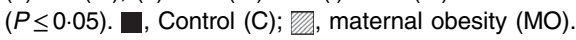


(a)
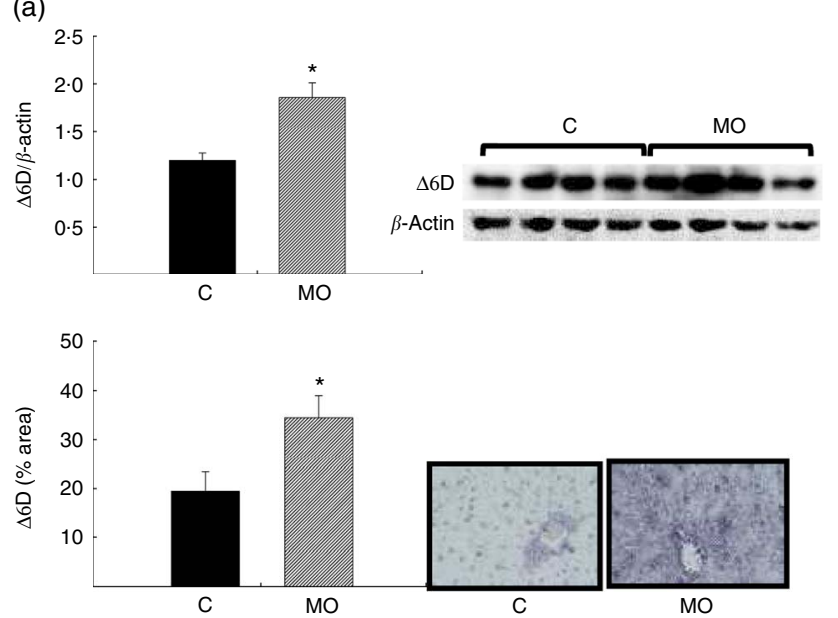

(c)
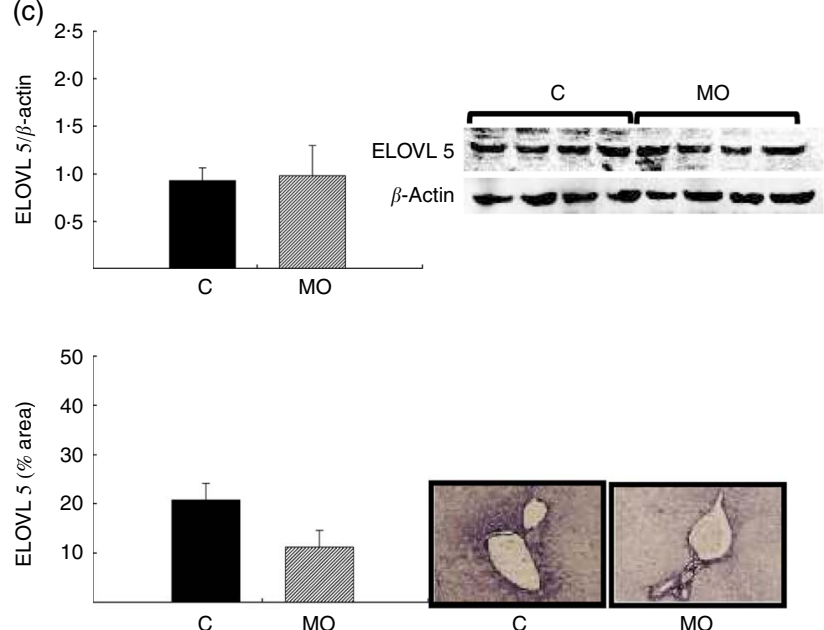

(b)
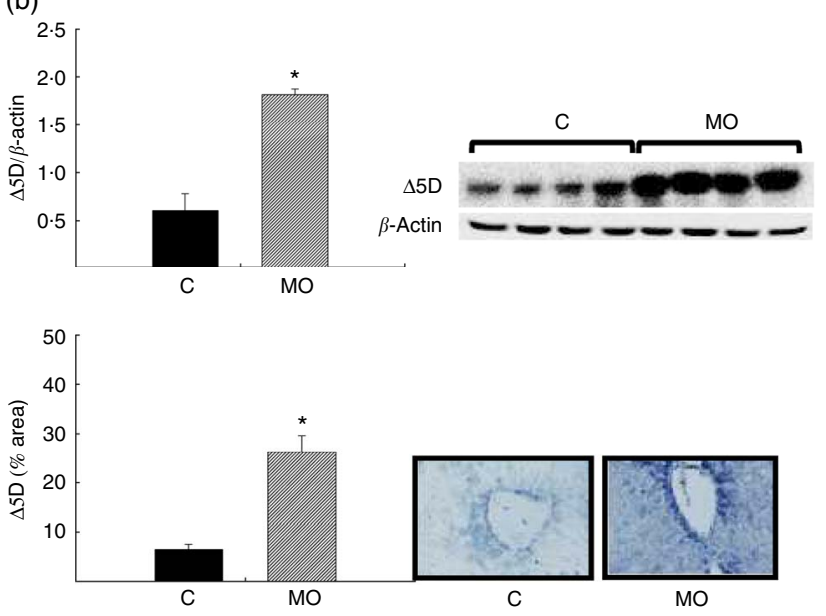

(d)
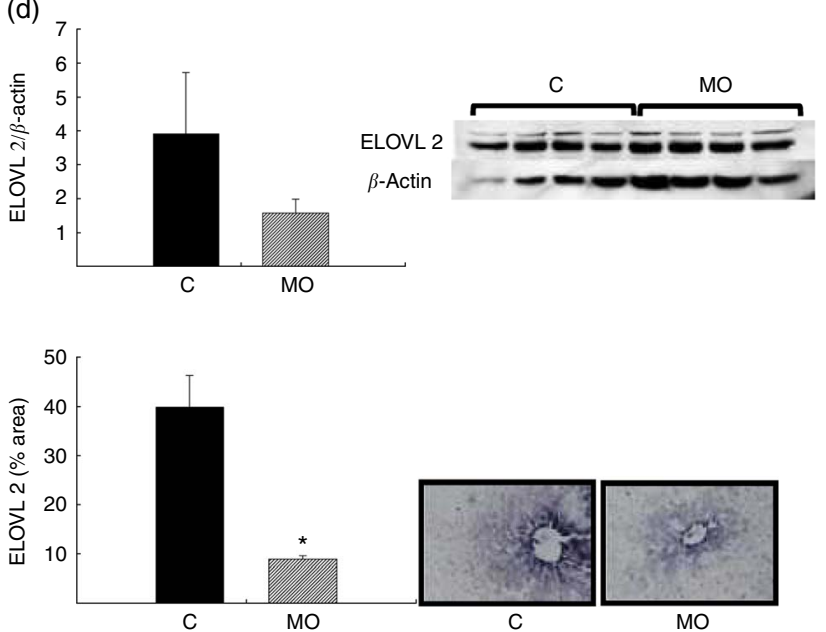

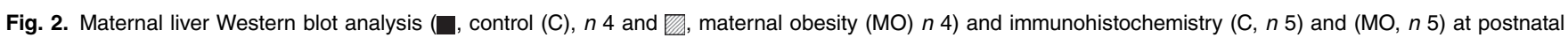
day 21. (a) Delta 6 desaturase $(\Delta 6 \mathrm{D})$, (b) delta 5 desaturase $(\Delta 5 \mathrm{D})$, (c) elongase 5 (ELOVL 5) and (d) elongase 2 (ELOVL 2). Values are means, with their standard errors represented by vertical bars. ${ }^{*}$ Mean value was significantly different $(P \leq 0.05)$.
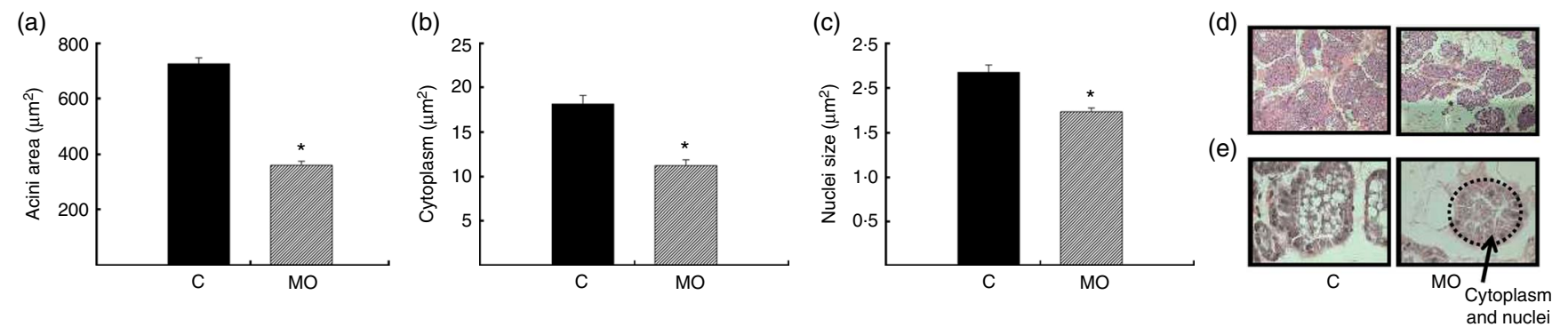

Fig. 3. Maternal mammary gland at postnatal day 21. (a) Acini area $\left(\mu \mathrm{m}^{2}\right)$, (b) cytoplasm $\left(\mu \mathrm{m}^{2}\right)$, (c) nuclei size $\left(\mu \mathrm{m}^{2}\right)$ and (d) microphotography at $10 \times$ the adipose tissue area in white and parenchymal tissue in black, and (e) microphotography at 100x cytoplasm and nuclei size. Values are means $(n 5)$, with their standard errors represented by vertical bars. ${ }^{*}$ Mean value was significantly different $(P \leq 0.05)$. $\square$, Control $(\mathrm{C})$; 覆, maternal obesity (MO).

as relative brain weight to body weight they were similar. At PND 36, brain weights were similar in $\mathrm{C}$ and MO female pups, whereas in male pups brain weight was lighter in MO than in $\mathrm{C}$ (Table 3). Brain weight relative to body weight was lower in male MO than in female MO. Female brain weight was lighter in comparison with male brain weight in the C offspring at PND 36.
There was no difference in liver weight at PND 21 and 36 between groups. However, liver weight in MO male pups was heavier than in MO female pups. Liver weight relative to body weight was lower in MO female pups compared with $\mathrm{C}$ and in $\mathrm{C}$ male pups compared with $\mathrm{C}$ female pups. Total liver fat and TAG were similar among groups and sexes (Table 3). 
(a)

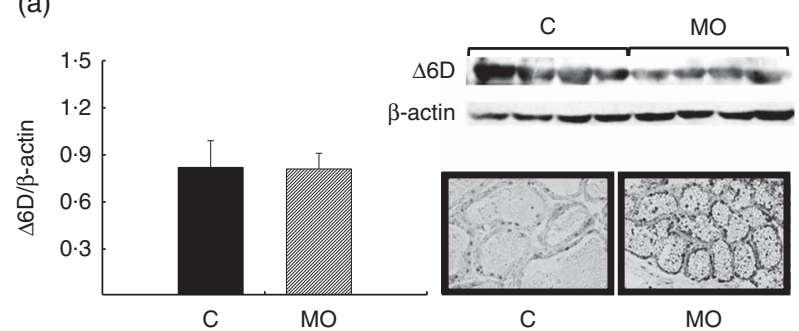

(c)

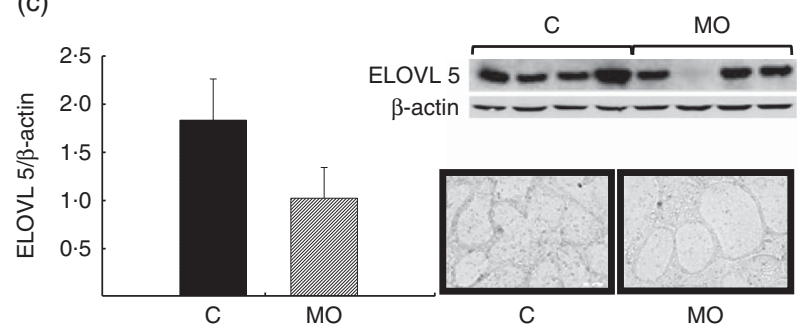

(b)

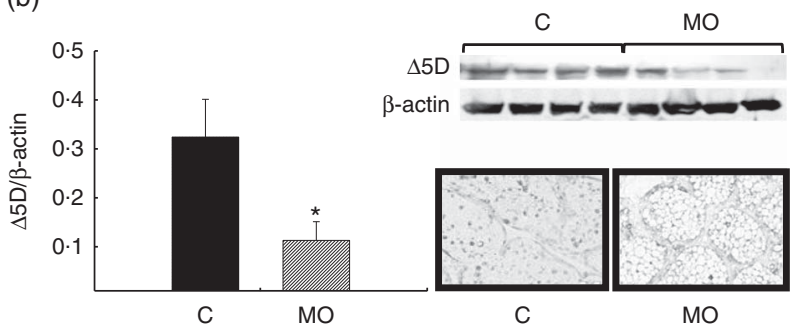

(d)

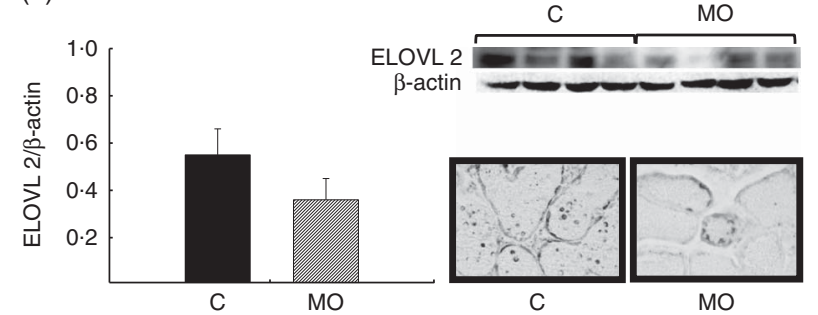

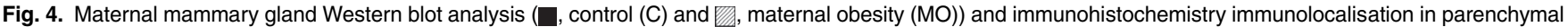
cells at postnatal day 21. (a) Delta 6 desaturase $(\Delta 6 \mathrm{D})$, (b) delta 5 desaturase $(\Delta 5 \mathrm{D})$, (c) elongase 5 (ELOVL 5 ) and (d) elongase 2 (ELOVL 2). Values are means $(n 4)$, with their standard errors represented by vertical bars. * Mean value was significantly different $(P \leq 0.05)$.

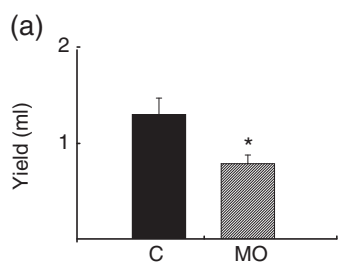

(b)

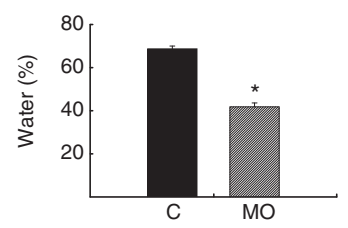

(f)

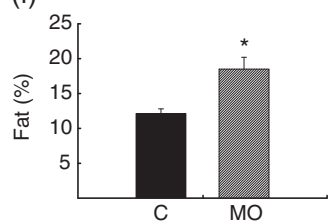

(g)

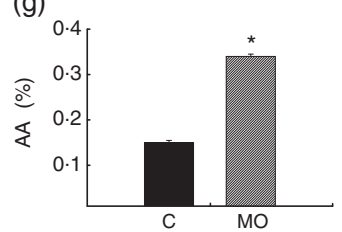

(c)

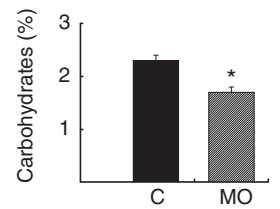

(h)

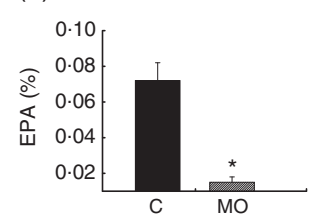

(d)

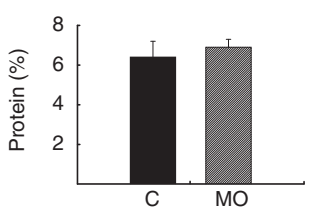

(e)

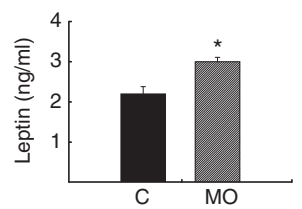

(j)

Fig. 5. Maternal milk components at postnatal day 20. (a) Total yield (ml), (b) water (\%), (c) carbohydrates (\%), (d) protein (\%), (e) leptin (ng/ml), (f) fat (\%), $(\mathrm{g})$ arachidonic acid (AA) (\%), (h) EPA (\%), (i) DHA (\%) and (j) percentage of fatty acid in milk. Values are means $(n 5)$, with their standard errors represented by vertical bars. * Mean value was significantly different $(P \leq 0.05)$. $\square$, Control $(C)$; T/r, maternal obesity $(\mathrm{MO})$.

\section{Discussion}

Milk protein, carbohydrates and lipids such as LC-PUFA are essential components for optimal offspring growth, brain development and maturation of the immune system. There is a clear association between maternal obesity and failure of milk production in human and animal studies ${ }^{(25-27)}$. However, effects of a high-fat diet consumption and maternal obesity on maternal liver and MG synthesis of enzymes related to milk production and composition are not well-documented. Our data show that maternal obesity adversely affects liver and MG function, resulting in decreased milk fatty acid quality, associated with negative effects in offspring development. We previously reported that female rats exposed to a $25 \%$ fat diet from their own weaning to adult life and through gestation and lactation show elevated insulin, glucose, homeostasis model assessment (HOMA), leptin, TAG and cholesterol at the end of their lactation period ${ }^{(3)}$. The present study shows that maternal obesity because of a high-fat diet affects maternal hepatic and MG function, alters milk nutrient concentration and negatively programmes offspring metabolism.

The liver has a critical role in fatty acid metabolism, including control of the synthesis and production of LC-PUFA during lactation $^{(17)}$. The fatty acids are then transported to the MG where they constitute key components of milk. Maternal highfat intake during lactation alters blood lipid concentrations and liver metabolism with consequences for maternal homoeostasis, oxidative stress, lipogenesis and $\beta$-oxidation ${ }^{(3,19,28-30)}$. In the present study, the maternal high-fat diet increased maternal liver fat without any effect on liver weight. Importantly, the 
Table 3. Pup parameters at birth and at postnatal day (PND) 21 and 36 (Mean values with their standard errors; $n 5$ litters)

\begin{tabular}{|c|c|c|c|c|c|c|c|c|}
\hline & \multicolumn{2}{|c|}{$C(n 5)$} & & & \multicolumn{2}{|c|}{$\mathrm{MO}(n 5)$} & & \\
\hline & Mean & SEM & & & Mean & SEM & & \\
\hline \multicolumn{9}{|l|}{ Pup characteristics at birth } \\
\hline Body weight at birth $(\mathrm{g})$ & $5 \cdot 6$ & 0.04 & & & $5 \cdot 4$ & 0.06 & & \\
\hline Gain weight during lactation (g) & 31.4 & 0.9 & & & $35 \cdot 6^{*}$ & 0.7 & & \\
\hline \multicolumn{9}{|l|}{ Pup characteristics at PND 21} \\
\hline Body weight $(\mathrm{g})$ & 37 & 1.8 & & & 41 & 1.4 & & \\
\hline Adipose tissue weight (g) & 0.4 & 0.01 & & & $0.7^{\star}$ & 0.006 & & \\
\hline Relative adipose tissue weight (\%) & 0.8 & 0.6 & & & $1 \cdot 6^{*}$ & 0.7 & & \\
\hline Brain weight $(\mathrm{g})$ & 1.4 & 0.02 & & & $1 \cdot 2^{*}$ & 0.02 & & \\
\hline Relative brain weight (\%) & 3.4 & 0.2 & & & 3.0 & 0.1 & & \\
\hline Liver weight $(\mathrm{g})$ & 1.4 & 0.01 & & & 1.3 & 0.05 & & \\
\hline \multirow[t]{2}{*}{ Relative liver weight (\%) } & $3 \cdot 4$ & 0.008 & & & $3 \cdot 1$ & 0.1 & & \\
\hline & \multicolumn{2}{|c|}{$\mathrm{C}+$} & \multicolumn{2}{|c|}{ MO } & \multicolumn{2}{|c|}{$\mathrm{Co}^{+}$} & \multicolumn{2}{|c|}{ MO } \\
\hline \multicolumn{9}{|l|}{ Pup characteristics at PND 36} \\
\hline Body weight $(\mathrm{g})$ & 117 & 5 & 122 & 1 & $139 \dagger$ & 7 & $141 \dagger$ & 3 \\
\hline Adipose tissue weight (g) & 0.7 & 0.1 & $1.0^{\star}$ & 0.04 & $1.0 \dagger$ & 0.12 & $1.5^{\star} \dagger$ & 0.37 \\
\hline Relative adipose tissue weight (\%) & 0.6 & 0.06 & $0.8^{\star}$ & 0.03 & $0.8 \dagger$ & 0.02 & $1.0 *+$ & 0.09 \\
\hline Brain weight $(\mathrm{g})$ & 1.6 & 0.02 & 1.6 & 0.02 & $1.8 \dagger$ & 0.02 & $1.6^{*}$ & 0.02 \\
\hline Relative brain weight (\%) & 1.5 & 0.08 & 1.3 & 0.02 & 1.3 & 0.07 & $1.2 \dagger$ & 0.02 \\
\hline Liver weight $(\mathrm{g})$ & 4.7 & 0.2 & 4.5 & 0.1 & 5.1 & 0.3 & $5.3 \dagger$ & 0.3 \\
\hline Relative liver weight (\%) & 4.1 & 0.05 & $3 \cdot 8^{\star}$ & 0.1 & $3.7 \dagger$ & $0 \cdot 1$ & 3.7 & 0.1 \\
\hline Liver total fat (\%) & 4.8 & 0.02 & 4.8 & 0.02 & 4.0 & 0.09 & 4.5 & 0.03 \\
\hline Liver TAG ( $\mathrm{g} / \mathrm{g}$ of liver) & 0.02 & 0.001 & 0.01 & 0.001 & 0.02 & 0.003 & 0.03 & 0.005 \\
\hline
\end{tabular}

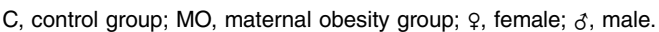

* Significantly different compared with $C(P \leq 0.05)$.

† Significantly different compared with male $(P \leq 0.05)$.

percentages of $n-3$ PUFA and n-6 PUFA and AA and EPA were lower in MO mothers, whereas SFA and monosaturates increased relative to $\mathrm{C}$ mothers. $\Delta 6 \mathrm{D}$ and $\Delta 5 \mathrm{D}$ were increased probably because of negative feedback regulation by decreased ELVOL 2 expression in MO mothers compared with $\mathrm{C}$ rats. Availability of milk nutrients is determined by the interaction of dietary intake, intestinal absorption and maternal metabolism in several tissues especially the liver and MG. There is evidence to indicate that desaturases are increased in a fatty liver ${ }^{(13,31-34)}$, which would explain the increased abundance of desaturases and elongases that we observed in the livers of the obese mothers. However, there is also evidence that low dietary PUFA increase liver desaturases and elongases ${ }^{(21)}$ and therefore in our study we might have expected these enzymes to decrease in obese mothers given their increased PUFA intake. A potential explanation of this apparent contradiction would be that it is not the total PUFA in the diet that regulates the hepatic production of these enzymes but the ratio of PUFA:SFA. In agreement with these findings, López-Vicario et al. ${ }^{(35)}$ produced non-alcoholic steatohepatitis in mice by feeding them with a high-fat diet resulting in an increase of liver $\Delta 5 \mathrm{D}$ and $n-6$ PUFA.

The relative proportions of cellular compartments change markedly during MG development in pregnancy and lactation. Adipose tissue is replaced by parenchymal tissue ${ }^{(27)}$. In our study, MG development in obese mothers did not produce the normal structure with resultant changes in MG function. In our study, MG development in obese mothers did not produce the normal pattern of relative development of parenchymal (acinar and ductal epithelium) and adipose tissues. MO groups showed more adipose and less parenchymal tissue compared with $\mathrm{C}$, likely to result in altered MG function. A high-fat intake produces similar outcomes in obese pregnant mice ${ }^{(36)}$, suggesting that maternal high-fat diet delays lobuloalveolar structure development and differentiation during lactogenesis affecting milk production. Recently, Saben et $a l .{ }^{(36)}$ observed that a high-fat diet in lactating mice impairs de novo fatty acid synthesis in the MG through inhibition of acetyl CoA carboxylase mediated by adenosine monophosphateactivated protein kinase; this protein is overexpressed during lactation when lipid production begins (Fig. 6).

Milk nutrients are derived from dietary intake and liver and MG biosynthesis ${ }^{(37-39)}$. Studies in rats indicate that hepatic desaturase and elongase expression is dependent on PUFA availability. High levels of desaturases are present in livers of rats fed a low-PUFA diet $^{(13)}$. During pregnancy and lactation, some metabolic adaptations occur. LC-PUFA are synthesised in the liver and other extrahepatic tissue such as the MG or they are mobilised from adipose tissue reserves ${ }^{(32)}$. In the rat, it has been shown that approximately $35 \%$ of dietary linoleic acid is transferred directly to the MG and $12 \%$ to the milk independently of the dietary lipid content. The authors suggest that both the MG and the liver have an important role in the synthesis of LC-PUFA in the milk ${ }^{(13,32)}$.

Leptin is present in the milk of many different species, including rats ${ }^{(11)}$ and humans ${ }^{(40)}$. Nursing rats transfer leptin to the neonates via the milk. This transfer may regulate neonatal food intake ${ }^{(41)}$. Milk leptin concentration is determined by a number of factors. In the present study, milk leptin concentration was higher in $\mathrm{MO}$ compared with $\mathrm{C}$, associated with higher fat depots in $\mathrm{MO}$ offspring.

Studies in rats programming model of $50 \%$ maternal energy restriction have analysed rat milk composition and found less water in the milk and more fat at days 10 and 21 of lactation, in addition to less carbohydrate at day 10 of lactation ${ }^{(39)}$ results very similar to those we report here. In our study, the milk composition changes 


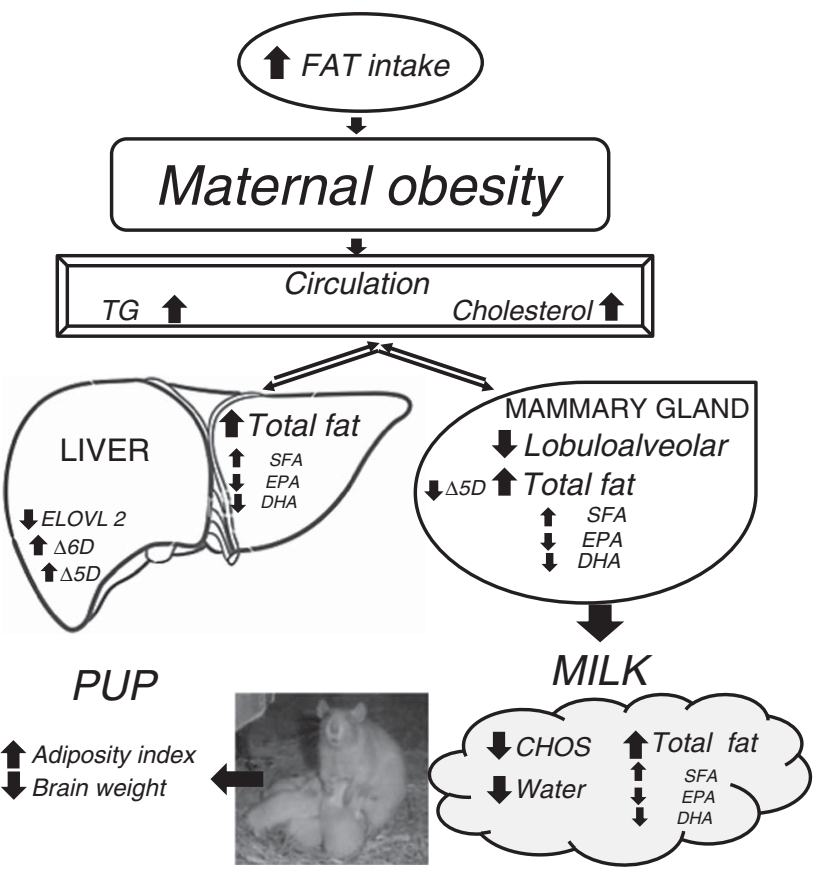

Fig. 6. Mechanisms proposed in rats fed an obesogenic diet during growth, pregnancy and lactation and effects on milk composition and offspring outcomes.

were associated with decreased brain weight and more larger fat depots in offspring at PND 21 and 36, supporting our reported findings of negative effects on adult metabolic function ${ }^{(42)}$, as well as cognitive function leading to behaviour impairment ${ }^{(22)}$. Absolute weight is the best overall indication of the total number of synapses and neurons, and clearly absolute function throughout life will be affected by having a smaller absolute brain size than controls. In addition, low milk DHA concentrations have been reported ${ }^{(14,16)}$ to affect offspring neural cell membranes and to induce damage in cognitive function during early life.

One very interesting sex-dependent observation in our study was that both absolute and relative brain weight decreased in male pups but not in female pups. These phenotypic changes are in keeping with our demonstration that $\mathrm{MO}$ increases offspring hippocampal reactive oxygen stress in adult life ${ }^{(43)}$. We have reported that MO offspring showed higher TAG, adipose tissue, leptin and insulin resistance at PND 21, 36 and $110^{(3,22)}$. Other groups have previously demonstrated that adequate lipid concentration in maternal milk is important for offspring organ maturation ${ }^{(44,45)}$.

In conclusion, our results indicate that maternal high-fat diet and obesity impair maternal liver and MG development and function, and modify milk composition, associated with dysregulated offspring brain development and metabolic function in a sex-specific manner.

\section{Acknowledgements}

This work was supported by Fundacion Mexicana para la Salud y Fundación Mexicana para la salud Hepatica (C. J. B., FUNSALUD and FUNDHEPA) and Consejo Nacional de Ciencia y Tecnología (CONACyT, E. Z., 155166 and C. J. B., 237643) Mexico. FUNSALUD, FUNDHEPA AND CONACyT had no role in the design, analysis or writing of this article.
C. J. B., researched data, responsible for the study design and manuscript writing; S. M., V. R., researched data; A. M., contributed to the discussion and reviewed the manuscript; P. W. N. and N. A. B., responsible for the study design and preparation of the manuscript; E. Z., responsible for the study design and preparation of the manuscript.

The authors declare that there are no conflicts of interest.

\section{References}

1. Zambrano E \& Nathanielsz PW (2013) Mechanisms by which maternal obesity programs offspring for obesity: evidence from animal studies. Nutr Rev 71, Suppl 1, S42-S54.

2. Waterland RA \& Garza C (1999) Potential mechanisms of metabolic imprinting that lead to chronic disease. Am J Clin Nutr 69, 179-197.

3. Vega CC, Reyes-Castro LA, Bautista CJ, et al. (2013) Exercise in obese female rats has beneficial effects on maternal and male and female offspring metabolism. Int J Obes (Lond) 39, $712-719$

4. Ke DS (2006) Using "number needed to treat" to interpret treatment effect. Acta Neurol Taiwan 15, 120-126.

5. Zhou H, Kong DH, Ma R, et al. (2006) Store-operated Ca2+ channels in rat colonic smooth muscle cells. Chin J Appl Physiol 22, 220-224.

6. Kong DH, Zhou H, Song J, et al. (2006) Capacitative $\mathrm{Ca}^{2+}$ entry is involved in ACh-induced distal colon smooth muscle contraction in rats. Acta Physiol Sin 58, 149-156.

7. Gocheva V, Zeng W, Ke D, et al. (2006) Distinct roles for cysteine cathepsin genes in multistage tumorigenesis. Genes Dev 20, 543-556.

8. Hall B (1975) Changing composition of human milk and early development of an appetite control. Lancet i, 779-781.

9. Grove KL, Allen S, Grayson BE, et al. (2003) Postnatal development of the hypothalamic neuropeptide $\mathrm{Y}$ system. Neuroscience 116, 393-406.

10. Knight CH, Alamer MA, Sorensen A, et al. (2004) Metabolic safety-margins do not differ between cows of high and low genetic merit for milk production. J Dairy Res 71, 141-153.

11. Bautista CJ, Boeck L, Larrea F, et al. (2008) Effects of a maternal low protein isocaloric diet on milk leptin and progeny serum leptin concentration and appetitive behavior in the first 21 days of neonatal life in the rat. Pediatr Res 63, 358-363.

12. Keen CL, Lonnerdal B, Clegg M, et al. (1981) Developmental changes in composition of rat milk: trace elements, minerals, protein, carbohydrate and fat. J Nutr 111, 226-236.

13. Rodriguez-Cruz M, Tovar AR, Palacios-Gonzalez B, et al. (2006) Synthesis of long-chain polyunsaturated fatty acids in lactating mammary gland: role of Delta5 and Delta6 desaturases, SREBP-1, PPARalpha, and PGC-1. J Lipid Res 47, 553-560.

14. Heird WC \& Lapillonne A (2005) The role of essential fatty acids in development. Annu Rev Nutr 25, 549-571.

15. Lauritzen L, Hansen HS, Jorgensen MH, et al. (2001) The essentiality of long chain $n-3$ fatty acids in relation to development and function of the brain and retina. Prog Lipid Res 40, 1-94.

16. Diau GY, Hsieh AT, Sarkadi-Nagy EA, et al. (2005) The influence of long chain polyunsaturate supplementation on docosahexaenoic acid and arachidonic acid in baboon neonate central nervous system. BMC Med $\mathbf{3}, 11$.

17. Vernon RG (2005) Lipid metabolism during lactation: a review of adipose tissue-liver interactions and the development of fatty liver. J Dairy Res 72, 460-469.

18. Ganapathy S (2009) Long chain polyunsaturated fatty acids and immunity in infants. Indian Pediatr 46, 785-790. 
19. Bell AW (1995) Regulation of organic nutrient metabolism during transition from late pregnancy to early lactation. J Anim Sci 73, 2804-2819.

20. Koletzko B, Agostoni C, Carlson SE, et al. (2001) Long chain polyunsaturated fatty acids (LC-PUFA) and perinatal development. Acta Paediatr 90, 460-464.

21. Rodriguez-Cruz M, Sanchez R, Sanchez AM, et al. (2011) Participation of mammary gland in long-chain polyunsaturated fatty acid synthesis during pregnancy and lactation in rats. Biochim Biophys Acta 1811, 284-293.

22. Rodriguez JS, Rodriguez-Gonzalez GL, Reyes-Castro LA, et al. (2012) Maternal obesity in the rat programs male offspring exploratory, learning and motivation behavior: prevention by dietary intervention pre-gestation or in gestation. Int $J$ Dev Neurosci 30, 75-81.

23. Torres N, Bautista CJ, Tovar AR, et al. (2010) Protein restriction during pregnancy affects maternal liver lipid metabolism and fetal brain lipid composition in the rat. Am J Physiol Endocrinol Metab 298, E270-E277.

24. Bautista CJ, Rodriguez-Gonzalez GL, Torres N, et al. (2013) Protein restriction in the rat negatively impacts long-chain polyunsaturated fatty acid composition and mammary gland development at the end of gestation. Arch Med Res 44, 429-436.

25. Turcksin R, Bel S, Galjaard S, et al. (2014) Maternal obesity and breastfeeding intention, initiation, intensity and duration: a systematic review. Matern Child Nutr 10, 166-183.

26. Amir LH \& Donath S (2007) A systematic review of maternal obesity and breastfeeding intention, initiation and duration. BMC Pregnancy Childbirth 7, 9.

27. Jevitt C, Hernandez I \& Groer M (2007) Lactation complicated by overweight and obesity: supporting the mother and newborn. J Midwifery Womens Health 52, 606-613.

28. Bertics SJ, Grummer RR, Cadorniga-Valino C, et al. (1992) Effect of prepartum dry matter intake on liver triglyceride concentration and early lactation. J Dairy Sci 75, 1914-1922.

29. Bell AW \& Bauman DE (1997) Adaptations of glucose metabolism during pregnancy and lactation. I Mammary Gland Biol Neoplasia 2, 265-278.

30. Kuhla B, Kucia M, Gors S, et al. (2010) Effect of a high-protein diet on food intake and liver metabolism during pregnancy, lactation and after weaning in mice. Proteomics 10, 2573-2588.

31. Niculescu MD, Lupu DS \& Craciunescu CN (2013) Perinatal manipulation of alpha-linolenic acid intake induces epigenetic changes in maternal and offspring livers. FASEB J 27, 350-358.

32. Rodriguez-Cruz M, Sanchez R, Bernabe-Garcia M, et al. (2009) Effect of dietary levels of corn oil on maternal arachidonic acid synthesis and fatty acid composition in lactating rats. Nutrition 25, 209-215.
33. Igal RA, Mandon EC \& de Gomez Dumm IN (1991) Abnormal metabolism of polyunsaturated fatty acids in adrenal glands of diabetic rats. Mol Cell Endocrinol 77, 217-227.

34. Nakamura MT \& Nara TY (2002) Gene regulation of mammalian desaturases. Biochem Soc Trans 30, 1076-1079.

35. López-Vicario C, Rius B, Morán-Salvador E, et al. (2014) Molecular interplay between $\Delta 5 / \Delta 6$ desaturases and long-chain fatty acids in the pathogenesis of non-alcoholic steatohepatitis. Gut 63, 344-355.

36. Saben JL, Bales ES, Jackman MR, et al. (2014) Maternal obesity reduces milk lipid production in lactating mice by inhibiting acetyl-CoA carboxylase and impairing fatty acid synthesis. PLOS ONE 9, e98066.

37. Anderson SM, Rudolph MC, McManaman JL, et al. (2007) Key stages in mammary gland development. Secretory activation in the mammary gland: it's not just about milk protein synthesis!. Breast Cancer Res 9, 204.

38. Varvikko T, Vanhatalo A, Jalava T, et al. (1999) Lactation and metabolic responses to graded abomasal doses of methionine and lysine in cows fed grass silage diets. J Dairy Sci 82, 2659-2673.

39. Wattez JS, Delmont A, Bouvet M, et al. (2015) Maternal perinatal undernutrition modifies lactose and serotranferrin in milk: relevance to the programming of metabolic diseases? Am J Physiol Endocrinol Metab 308, E393-E401.

40. Houseknecht KL, McGuire MK, Portocarrero CP, et al. (1997) Leptin is present in human milk and is related to maternal plasma leptin concentration and adiposity. Biochem Biophys Res Commun 240, 742-747.

41. Casabiell X, Pineiro V, Tome MA, et al. (1997) Presence of leptin in colostrum and/or breast milk from lactating mothers: a potential role in the regulation of neonatal food intake. J Clin Endocrinol Metab 82, 4270-4273.

42. Zambrano E, Martinez-Samayoa PM, Rodriguez-Gonzalez GL, et al. (2010) Dietary intervention prior to pregnancy reverses metabolic programming in male offspring of obese rats. J Physiol 588, 1791-1799.

43. Reyes Castro LA, Rodrigues Gonzalez GL, Larrea F, et al. (2014) Maternal resveratrol before and in pregnancy decreases hippocampal reactive oxygen stress and improves associative learning in male rat offspring of mothers fed an obesogenic diet. SGI 21, 365A (S-094).

44. Innis SM (2004) Polyunsaturated fatty acids in human milk: an essential role in infant development. Adv Exp Med Biol 554, $27-43$.

45. Cetin I \& Koletzko B (2008) Long-chain omega-3 fatty acid supply in pregnancy and lactation. Curr Opin Clin Nutr Metab Care 11, 297-302. 